\title{
Questes
}

Revue pluridisciplinaire d'études médiévales

Journée d'étude 1 - Trier, classer, organiser | 2022

Trier, classer, organiser

\section{Écrire e(s)t ordonner : deux exemples littéraires autour de l'arbor}

Sergi Sancho Fibla

\section{(2) OpenEdition}

10 Journals

Édition électronique

URL : https://journals.openedition.org/questes/5967

DOI : 10.4000/questes.5967

ISSN : 2109-9472

Éditeur

Les Amis de Questes

Édition imprimée

Pagination : 85-104

ISSN : 2102-7188

Référence électronique

Sergi Sancho Fibla, «Écrire e(s)t ordonner : deux exemples littéraires autour de l'arbor », Questes [En ligne], Journée d'étude 1 - Trier, classer, organiser | 2022, mis en ligne le 31 janvier 2022, consulté le 21 février 2022. URL : http://journals.openedition.org/questes/5967; DOI : https://doi.org/10.4000/ questes.5967

\section{(C) Association des amis de «Questes »}




\title{
Écrire e(s)t ordonner : deux exemples littéraires autour de l'arbor
}

\author{
Sergi SANCHO FIBLA
}

Aix-Marseille Université*

Le but de cet article est de montrer, à partir de l'usage littéraire des structures arborescentes, la manière dont l'acte - ou les actes - d'écriture put être, au bas Moyen Âge, une pratique liée à la notion contemporaine d'ordre et de classification. En effet, dans cette période de développement de différents types d'écriture, ces deux notions-l'ordre et la classification - avaient des marges sémantiques difficiles à définir ${ }^{1}$. Afin de mieux comprendre cet exercice dans le contexte médiéval tardif, il faudrait d'abord abandonner certains préjugés de la critique littéraire moderne qui nous empêchent de saisir la dimension anthropologique des pratiques d'écriture et de lecture ${ }^{2}$.

En ce sens, l'un des multiples aspects à considérer à propos de l'écriture médiévale est son rapport avec la visibilité du texte ${ }^{3}$. Parmi les

\footnotetext{
* La version initiale de cet article a été rédigée en 2017 à la suite des journées d'étude. ${ }^{1}$ Pour la grande variété des contextes et types d'écriture des derniers siècles du Moyen Âge, voir : Médiévales, $n^{\circ}$ 56, dir. Étienne Anheim et Pierre Chastang, 2009 ; et Paul Bertrand, Les Écritures ordinaires. Sociologie d'un temps de révolution documentaire (entre royaume de France et Empire, 1250-1350), Paris, Publications de la Sorbonne, 2015.

${ }^{2}$ Mary Carruthers, The Book of Memory: A Study of Memory in Medieval Culture, Cambridge, Cambridge University Press, 1990, p. 1.

${ }^{3}$ Cette question a constamment été revue pendant les dernières décennies. Voir, par exemple, L'Image: fonctions et usages des images dans l'Occident médiéval, actes $d u \sigma^{e}$ "International workshop on medieval sociétés», Centre Ettore Majorana, Erice, Sicile, 17-23 octobre 1992, dir. Jérôme Baschet et Jean Claude Schmitt, Paris, Le Léopard d'or, 1996 ; Olivier Boulnois, Au-delà de l'image. Une Archéologie du visuel au Moyen-Âge V-XVI siècle, Paris, Seuil, 2008 ; The Mind's Eye. Art and
} 
différentes approches relatives à la nature visuelle de la lecture et de l'écriture, un élément nous intéresse particulièrement. Il s'agit des outils de rhétorique visuelle et d'exégèse, qui n'avaient pas seulement une fonction didactique, mais participaient directement à la création et à la réception des textes ${ }^{4}$. Les structures diagrammatiques en particulier étaient des mécanismes cognitifs qui avaient un rôle remarquable dans l'organisation et le développement des arguments d'un exposé oral ou écrit. Dans la plupart des cas, il était question d'images ou de schémas qui, suivant les préceptes de la rhétorique, fournissaient une structure au texte et procuraient un ordre pour l'exposition du contenu tout en facilitant - par leur reconnaissance et leur nature démonstrative - non seulement la lecture, mais aussi l'herméneutique.

Certaines de ces figures étaient considérées comme des arbres et offraient aux auteurs des armatures pour spatialiser et hiérarchiser les arguments suivant les parties plus ou moins figuratives d'un végétal : le tronc, les racines, les branches, etc. Mais l'arbre et l'arborescence sont des formes visuelles qui, à la fin du Moyen Âge, participent à de nombreuses sortes de manifestations iconographiques. Face à cette configuration graphique fortement polysémique, Naïs Virenque a réalisé une différenciation, que nous jugeons cruciale, entre l'image de «l'arbre répartiteur » et le « graphe arborescent ».

Theological Argument in the Middle Ages, dir. Jeffrey Hamburger, Anne-Marie Bouché, Princeton, Princeton University Press, 2006.

${ }^{4}$ À propos de la notion d'exégèse visuelle, voir notamment Anna C. Esmeijer, «Divina quaternitas »: A Preliminary Study in the Method and Application of Visual Exegesis, Assen/Amsterdam, Gorcum, 1978 ; Patrice Sicard, Diagrammes médiévaux et exégèse visuelle. Le "Libellus de formatione arche » de Hugues de Saint-Victor, Paris/Turnhout, Brepols, 1993 ; Christian Heck, «L'allégorie dans l'art médiéval : entre l'exégèse visuelle et la rhétorique de l'image », dans L'Allégorie dans l'art du Moyen Âge. Formes et fonctions. Héritages, créations, mutations, dir. Christian Heck, Turnhout, Brepols, 2011, p. 7-22. 
Dans le premier cas, on y trouve une figure d'un arbre pourvu de tronc et branches et/ou fleurs et/ou fruits. Ces éléments forment les lieux pour répartir l'information :

De tels arbres se caractérisent par leur continuité formelle et figurative: leur structure englobe leurs contenus, de sorte que chaque élément qu'ils comportent se trouve à l'intérieur d'un cadre localisant. La plupart du temps, elles répartissent des contenus finis tous les éléments d'une liste, l'intégralité d'un système de correspondances ou au contraire une sélection volontairement limitée de textes, de scènes ou de personnages ${ }^{5}$.

De l'autre côté, le «graphe arborescent» serait une figure de caractère stemmatique dépourvue de cadres qui localisent les informations. Par conséquent, elle ne montre pas la continuité formelle et figurative entre les éléments graphiques d'un arbre - tronc, racines, feuilles, branches, fruits - et les éléments que ces parties organisent :

Bien que les graphes arborescents donnent à voir le plan d'une arborescence de manière parfois très éloignée d'une imitation de l'arbre, ils opèrent comme les images d'arbres répartiteurs : la seule différence réside dans la présence ou dans l'absence de cadres localisants ${ }^{6}$.

Contrairement aux arbres, ces graphes arborescents sont non limités et peuvent être ouverts à la prolongation-matérialisée ou mentale - des contenus présentés dans les subdivisions. Ainsi, «alors que l'arbre donne à voir un ordre, l'arborescence donne à voir un processus ordonné $»^{7}$. Ces outils, arbres et arborescences, constituaient un répertoire

\footnotetext{
${ }^{5}$ Naïs Virenque, Structures arborescentes et arts de la mémoire. Art, science et dévotion dans les ordres mendiants en France et en Italie du XIII ${ }^{e}$ au XVI ${ }^{e}$ siècle. Thèse dirigée par Maurice Brock, Universtié de Tours, 2019, p. 64.

${ }^{6}$ Ibid., p. 65.

${ }^{7}$ Ibid.,p. 64.
} 
commun de moyens d'expression et de transmission de la connaissance. Présents dès la phase d'inventio et de dispositio, ils permettaient à l'auteur de mettre en place les idées et de créer ou de développer, souvent à travers une logique combinatoire, de nouveaux contenus ${ }^{8}$. Ils permettaient au lecteur d'identifier les structures et d'en acquérir les connaissances. Enfin, tout comme l'auteur, le lecteur pouvait ensuite développer ses propres réflexions et les insérer dans ces figures: les avantages de cette structure, à savoir son caractère mnémotechnique et sa hiérarchie arborescente, incitaient à la combinaison et à la recombinaison. Ces arbores classificateurs sont considérés comme un répertoire commun entre auteur et lecteur, au point qu'ils pouvaient parfois disparaître de la page, devenant une image implicite tout en demeurant clairement compréhensible $^{9}$. Ainsi, les structures arborescentes ne devaient pas forcément être représentées graphiquement dans les manuscrits, mais pouvaient être suggérées implicitement par le texte. Elles constituaient alors ce que Mary Carruthers appelle des literary pictures $^{10}$, c'est-à-dire, des images littéraires qui guidaient le progrès du lecteur à travers un texte $^{11}$. Ainsi, comme Christine Klapitsch-Zuber l'a montré, au-delà de

\footnotetext{
${ }^{8}$ Mary Carruthers, «Mechanisms for the transmission of culture: the role of "place" in the arts of memory »dans Translatio, the Transmission of Culture in the Middle Ages, dir. Laura Hollengreen, Turnhout, Brepols, 2008, p. 1-26, cit. p. 3.

9 «Il importe d'appréhender la culture visuelle du Moyen Âge comme une culture à la fois matérielle et immatérielle, autrement dit comme une culture qui relève autant de la fréquentation d'images concrètes, palpables, que de l'invention, de la création, de l'imagination ou encore de la remémoration d'images mentales que peuvent susciter la visualisation d'une image matérielle, la lecture d'un texte ou l'écoute d'une musique ». Naïs Virenque, Ouv. cit., p. 66.

${ }^{10}$ Mary Carruthers, The Craft of Thought: Meditation, Rhetoric and the Making of Images, 400-1200, Cambridge, Cambridge University Press, 1998, p. 22.

${ }^{11}$ Un cas paradigmatique de ce procédé se trouve dans le Lignum Vitae de Bonaventure de Bagnoregio, qui invite le lecteur à imaginer un arbre («describe [...] in spiritu mentis tuae arborem quandam » ayant douze rameaux. Chaque rameaux est pourvu, à son tour, d'un fruit qui contient un épisode de la vie de Christ, qui est facilement mémorisable grâce à cette configuration graphique et mentale («propter facilitatem memoriae »). Saint Bonaventure, Lignum vitae, trad. André Ménard, Bron, Éditions en ligne des Frères Mineurs Capucins de la Province de France, 2011, 3,
} 
leur caractère mental ou matérialisé, ces mécanismes étaient un outil dans les processus de réflexion, d'exposition et d'exégèse dans des domaines très différents :

les arbores sont, avec d'autres figures, érigés en moyens de connaissance, à la fois didactiques et herméneutiques $[\ldots]$ et ces figures, mentales ou matérialisées, n'ont pas seulement valeur pédagogique. Leurs auteurs encouragent les élèves à réfléchir sur elles parce qu'ils sont convaincus que ces figures peuvent mieux approfondir une idée qu'un exposé discursif. L'image devient au $\mathrm{XII}^{\mathrm{e}}$ siècle un instrument d'exégèse autonome ${ }^{12}$.

Nous nous proposons ici d'analyser deux corpus de documents épistolaires dans lesquels la disparition matérialisée de ces formes coïncide avec l'émergence d'une structure arborescente dans les stratégies de rhétorique visuelle typique de l'écriture du XIVe siècle. Le premier corpus est constitué de deux lettres de Marguerite d'Oingt, chartreuse de la seconde moitié du XIII ${ }^{\mathrm{e}}$ et du début du XIV siècle, conservées dans le manuscrit contenant la totalité de ses œuvres ${ }^{13}$. Une

p. 3-4. Voir aussi Naïs Virenque, «Chapitre 4. 1. A. Imaginer un arbre pour méditer : la tradition mnémonique de l'ékphrasis» et «Chapitre 4. 2. B. Façonner un arbre dans la mémoire : le procédé littéraire de l'injonction au lecteur », dans Ouv. Cit., p. 274-301; et Michelle Karnes, Imagination, Meditation, and Cognition in the Middle Ages. Chicago, University of Chicago Press, 2011, p. 128.

12 Cf. Christine Klapisch-Zuber, L'Ombre des ancêtres. Essai sur l'imaginaire médiéval de la parenté, Paris, Fayard, 2000, p.43. Les arbores ont connu un développement très important à partir $\mathrm{du}_{\mathrm{XIII}}^{\mathrm{e}}$ siècle comme moyen de création et transmission de la connaissance. Toutefois, ces outils n'ont pas été figés dans le temps et donc des évolution et changements sont évidents dans les siècles successifs. Parmi les aspects intéressants de ces changements au cours du XIV e siècle : le processus de végétalisation progressif qui leur confère de plus en plus les caracteristiques visuelles d'un arbre, et l'homogeneïsation progressive des modèles, grâce, spécialement, à la diffusion que les ordres mendicants en firent à travers l'Europe. Ibid., p. 209-227 ; Michael Evans, «The Geometry of the Mind », Architectural Association Quarterly, 12, 4, 1980, p. 32-55 ; Naïs Virenque, Ouv. cit., p. 410-422.

${ }^{13} \mathrm{Ce}$ document est daté de la moitié du XIV ${ }^{\mathrm{e}}$ siècle. Il est conservé à la Bibliothèque de Grenoble sous la cote 5785R. Le manuscrit a été édité en 1965: Marguerite d'Oingt, Les Euvres de Marguerite d'Oingt, éd. Antonin Duraffour, Pierre Gardette, 
des missives illustre l'application d'un modèle arborescent qui évoque une spatialisation des cinq sens répondant au schéma dualiste chrétien. Cette disposition permet de comprendre un autre texte de la même auteure où la logique arborescente n'est qu'implicite. Le deuxième corpus est constitué également de deux lettres, écrites par Pétrarque et datées de la moitié du XIV ${ }^{\mathrm{e}}$ siècle. Il s'agit des épîtres L et LXI qui se trouvent dans Epistolae variae, un ensemble non organique de 76 compositions de différentes périodes traitant de sujets variés. Pétrarque avait exclu ces textes des lettres officielles et ils ne furent conservés que grâce à l'entremise d'admirateurs du poète. Ces deux lettres, toutes deux destinées à Giovanni Fedolfi da Parma et à son ami, Lucchino del Verme, témoignent d'un dialogue au cœur duquel le sujet principal est la réception d'une de ces structures littéraires arborescentes : d'après ces lettres, il semble que les interlocuteurs du poète aient identifié un modèle, envisagé son exact contraire et créé un troisième modèle issu de la combinaison des deux premiers.

\section{L'arbre sensoriel de Marguerite d'Oingt}

Marguerite d'Oingt était prieure du monastère de Poleteins autrefois situé dans la Dombes -, lorsqu'elle rédigea la première lettre, destinée à une moniale anonyme. Le texte commence comme un récit qui s'articule autour du mot vehemens ${ }^{14}$. La méditation que la narratrice entreprend autour de cette notion déclenche chez elle une vision d'un

et Paulette Durdilly, Paris, Société d'édition Les Belles Lettres, 1965, p. 144-147. Une version que nous ne suivrons pas dans cette étude, puisque la Bibliothèque de Grenoble permet aujourd'hui l'accès en ligne ouvert au manuscrit : http://pagella.bmgrenoble.fr/BMG.html?id=Bmg-0004773.

${ }^{14} \mathrm{La}$ lettre occupe les folios f. 33r-34r du ms. 5785 R. Ici, f. 33v, 1. 7-8. Quant à l'importance du mot "vehemence " dans l'articulation entre parole et vision, voir Sergi Sancho Fibla, Escribir y meditar. Las obras de Marguerite d'Oingt, Madrid, Siruela, El Árbol del Paraíso 93, 2018, p. 280-369. 
paysage désertique où s'élève une montagne avec un arbre sur son flanc $^{15}$. Ses cinq branches, désignant les cinq sens, se trouvent inclinées vers la terre tout comme ses racines. Sur sa cime, un obstacle empêche le soleil et la rosée de le nourrir. Alors, la vision s'anime par la description d'un violent impact : un torrent d'eau surgit, renverse l'arbre qui retrouve sa verdure alors que ses racines et ses branches, contenant les sens, se couvrent de feuilles et tendent, ensemble, vers le ciel ${ }^{16}$.

Cet arbre merveilleux est présenté au lecteur comme ayant un rapport explicite avec les sens et par extension, avec la condition humaine. D'une part, l'image de l'arbre-corps pourrait être associée aux représentations ovidiennes des métamorphoses, des arbres de consanguinité, des analogies lexicales des traités médicaux ou botaniques du Moyen Âge ou encore, de tous les motifs anthropo-végétaux présents chez d'autres auteurs de la Renaissance ${ }^{17}$. D'autre part, on pourrait penser à première vue que ce récit fait écho de l'image néoplatonicienne décrivant l'homme comme un végétal renversé et enraciné dans le ciel, cet aspect rappelant son appartenance originelle aux régions célestes ${ }^{18}$.

15 «En cel arbre aveit cinc branches que estoyent totes seches et totes enclinavunt ver terra. Et es feuylles de la premere branche avoyt escrit "visu". En la seconde avoyt escrit "aud[34r]itu". En la tierci avoy escrit "gustu". En la quarta avoyt escr[i]t "odoratu". En la cinquiesma avoyt escrit "tactu". Sus la cime de l'arbre avoyt un $\mathrm{g} r$ [a]nt rondel, come se fut un fonz de vayssel, si que li arbres estoyt toz clos par desus en tel maneri que li selouz en la rosee en poyent ferir per desus ». Ms. 5785R, $33 \mathrm{v}-34 \mathrm{r}$.

${ }^{16}$ Ele leva ses euz sus la mo[n]taygne et vit un grant ruysel qui descendit assi tres grant forci, que co semblavet una mer. Cele yeve [chisi] si tres duremant au pre de cel arbre que les ragies se viraront totes desus et la cime se metit en terra et les branches ver lo ciel. Et les foylles que erant totes seches furont totes reverdies, les ragies que erant devant fichies en terra furont totes espandues et drecies ver lo ciel et foront totes reverdies et follyes en maneri et de branches ». Ibid., $34 \mathrm{r}$.

17 Voir Corrado Confalonieri, « Alle radici dell'uomo-pianta. Fonti classiche per Dante, Boccaccio, Ariosto e Tasso », Campi immaginabili, $\mathrm{n}^{0}$ 38-39, vol. 1-2, 2008, p. 24-36.

${ }^{18}$ Image présente dans le Timée de Platon, mais aussi dans plusieurs textes médiévaux. Pour une liste plus exhaustive des références de cette analogie dans la littérature et l'art du Moyen Âge, voir Sergi Sancho Fibla, Quando bene respicio. 
Néanmoins, ni l'un ni l'autre ne semblent s'adapter au modèle présenté par Marguerite d'Oingt. En effet, le premier cas opère une analogie par isomorphie physique - feuillage et cheveux, par exemple - ou par fonction - sang et sève - qui n'a pas lieu chez la moniale chartreuse puisque le lien entre homme et végétal est strictement manifesté par la sensorialité. Pour ce qui est de l'image néoplatonicienne, elle présente une figure d'arbre renversé à laquelle l'homme devrait tendre puisque les racines-tête doivent se diriger vers le ciel. Mais l'image de Marguerite d'Oingt ne répond pas non plus à cette logique, puisqu'elle ne décrit pas la représentation naturaliste de l'arbre. De ce fait des chercheurs se sont interrogés sur la position de l'arbre, planté ou renversé. Pourtant nous pouvons considérer qu'en réalité elle importe peu car il s'agit d'un renversement symbolique : les racines et les branches tournent au même moment sans fournir de référence à l'ensemble du végétal ${ }^{19}$.

En revanche, cette description pourrait être liée au double diagramme de l'arbor et plus concrètement, au motif de l'arbor bonalarbor mala ${ }^{20}$. Il s'agit d'une représentation fondée sur la pratique de l'exégèse biblique qui est rapidement devenue une structure vide utilisée pour présenter des idées opposées. Loin de constituer un modèle incluant

Palabra, imagen y meditación en las obras de Marguerite d'Oingt, thèse doctorale sous la direction de Victoria Cirlot, Barcelona, Universitat Pompeu Fabra, 2015, p. 545-556.

${ }^{19}$ Nous retrouvons cette ambiguïté dans d'autres écrits de cette époque comme dans le chant XXII du Purgatoire de la Divine Comédie (vv.132-135). Dante parle d'un sapin qui se dégrade du haut vers le bas, description qui a donné lieu à de multiples interprétations textuelles ou illustrées sur la position de l'arbre : planté, inversé ou avec la cime inversée mais le tronc au-dessous. Voir Sergi Sancho Fibla, Quando bene respicio, op. cit., p. 586-594.

${ }^{20}$ Voir Hélène Toubert, «Une fresque de San Pedro de Sorpe (Catalogne) et le thème iconographique de l'Arbor bona-ecclesia, arbor mala-synagoga», Cahiers Archéologiques, vol. 19, 1970, p. 167-189 et Marlène Delsouiller, « Images de l'arbre sans feuilles des cathédrales de Tolède et de Barcelone (XIV $-\mathrm{XV}^{\mathrm{e}}$ siècles) », dans L'Arbre au Moyen Âge, dir. Valérie Fasseur, Danièle James Raoul, Jean-René Valette, Paris, Presses Universitaires de Paris-Sorbonne (Culture et civilisations médiévales, 49), 2010, p. 85-99. 
un contenu fixe, elle est une forme adoptant un ordre dialectique qui peut être dotée de différents messages : vices et vertus, synagogue et ecclesia, ou encore chute et rédemption ${ }^{21}$. La célèbre enluminure du Liber Floridus de Lambert de Saint-Omer, par exemple, ferait parfaitement écho à cette figure (fig. 1). Ce diagramme présente l'inversion spéculaire des deux arbres, disposée sur une double page de manuscrit. Les deux dessins naissent de la reliure des pages, l'un se développant à partir de la vertu principale, la caritas, tandis que l'autre le fait à partir de la cupiditas id est auaritia. Le feuillage abondant et les médaillons colorés de l'arbor bona s'opposent ainsi au caractère stérile et fané de l'arbor mala. La distribution symétrique des arbres établit une hiérarchie visuelle qui aide à la mémorisation, alors que la disposition parallèle des médaillons et leur contenu incitent le spectateur-lecteur à réfléchir, comparer et établir des connexions entre les deux ${ }^{22}$.

Marguerite d'Oingt semble trouver dans l'image de l'arbre des vices et des vertus, un outil pour façonner son expérience personnelle de la grâce ou de la puissance sensorielle de l'expérience divine. Au-delà de la structuration des végétaux, l'écriture des sens dans les feuilles des branches pourrait être une référence claire à ce type de diagramme. Mais la force de cette configuration graphique ne s'arrête pas là. L'exposition arborescente trouve son explication herméneutique dans la lettre suivante de ce manuscrit. En effet, le premier texte se termine de manière abrupte, sans commentaire ni glose morale de la part de l'auteur. Au contraire, la lettre qui suit est composée d'une vision et d'une explication finale qui

\footnotetext{
${ }^{21}$ Voir Adolf Katzenellebogen, Allegories of the Virtues and the Vices in Medieval Art. From Early Christian Times to the Thirteenth Century, Toronto, University of Toronto Press, 1989.

${ }^{22}$ Susanne Wittekind, «Visualizing Salvation: The Role of Arboreal Imagery in the Speculum Humanae Salvationis (Kremsmünster, Library of the Convent, Cod. 243) », dans, The Tree, Symbol, Allegory, and Mnemonic Device in Medieval Art and Thought, dir. Pippa Salonius et Andrea Worm, Turnhout, Brepols, 2014, p. 117-142, ici 123.
} 
suivent la même logique et qui pourraient faire penser qu'il s'agit de deux textes reliés. C'est en tout cas ce que nous envisageons dans cette étude ${ }^{23}$. L'auteur décrit cinq lumières suspendues dans le ciel à la suite desquelles le corps du Christ émerge et ses plaies se superposent aux cinq clartés ${ }^{24}$. À la fin de cette vision, le narrateur exhorte le lecteur à méditer sur les différentes parties du corps christique. Toutefois, cette énumération se structure de manière quintuple: les cinq plaies du corps du Sauveur, telles des branches, sont associées à cinq parties de son visage, correspondant à leur tour, aux cinq sens ${ }^{25}$.

Le motif des cinq branches de la première lettre se retrouve donc dans l'écriture du second texte où il devient un outil classificateur des prières grâce à l'association, présente dans les deux textes, du corps et de l'arbre sensoriel ou de l'arbre de la croix. Ainsi, dans le premier écrit,

${ }^{23}$ Outre les arguments ici exposés qui relient le contenu des deux textes, il faut souligner un autre indice présent dans le manuscrit qui soutient cette hypothèse. Alors que toutes les autres lettres de ce document sont séparées par la rubrique en rouge «Item alia epistola », il n'existe pas de phrase ni d'espace de rupture entre ces deux épîtres. Ms. 5785R, f. 34r.

${ }^{24}$ Ibid., 34v.

25 «Et proposa en son cuer que ele diroyt toz jors mais .1. pater noster el non de la passion Jhesu $\mathrm{Cr}[\mathrm{i}] \mathrm{t}$ et de ses beney[35r]tes playes et ordena cest pater noster en tel manere que ele en disoyt.V. en honour de son benoyt chie et de ceus benoyz cheveuz qui por nos furont si delava et en paignie. Et apres en disoyt autres .v. el nun de sos beneyz euz por co que il la regardat en pidie. Apres en dysoyt .v. en nun de ses douces oreylles qui tant orent de reproches por nos. Apres en disoyt .v. en honour de son benoyt nas per quoy il li donat sentir aucunes choses de sa tres grant doucour per la quele ele lo sout amer tendrement. Apres en disoit .v. en nun sa benoyte boche per quoy il li donat sa benicion et la appelat en son regno. Apres en disoyt el nun de la play del flan por ce qui el la voucist laver et bateyer de cele benoyte fontayne qui li sallit del flan. Apres illi disoyt .v. per chacuna mayn por ce qui el la vousit garder et deffendre en la force de ses braz de les mains a ses enemis. Apres dysoyt autres .v. por chacune playe des piez por ce que Jhesu Crit li perdonat ses pechiez ausi come il fit a la Magdalena ». Ibid., f. 34v-35r. Le toucher est le sens le plus ambigu dans cette association, mais il s'agit d'un aspect récurrent dans ce genre de correspondances entre le corps du Christ et les sens. Voir Sergi Sancho Fibla, "Penitential and Mystical senses. Two paths for Female Devotion in the Late Middle Ages », dans Sensual and Sensory Experiences in the Middle Ages. On Pleasure, Fear, Desire and Pain, dir. Carme Muntaner, David Carrillo-Rangel, Delfi Nieto-Isabel, Pau Castell,Newcastle upon Tyne, Cambridge Scholars Publishing, 2017, p. 141-158. 
l'inversion symétrique et la possibilité de combinaison permettent l'expression ou le façonnage de l'expérience, alors que dans le second texte le modèle est interprété et développé pour donner une projection idéale des cinq sens spirituels. De toute évidence, des arguments théologiques entérinent ces analogies entre les cinq sens et les parties du corps christique, en y associant le célèbre thème des sens spirituels et corporels dans la pensée mystique ${ }^{26}$. Toutefois, notre intérêt se porte surtout sur la forme d'organisation de ce rapport et sur la volonté de l'auteure de structurer sa méditation à travers une configuration arborescente explicite de la vision de soi et implicite de celle du Christ.

\section{L'arbre estival de Pétrarque}

Le second corpus se compose de deux lettres que Pétrarque envoie à Gênes à Giovanni Fedolfi da Parma et Lucchino del Verme. La première lettre, écrite durant l'été 1355, expose un argument apparemment assez prosaïque puisque la question posée est celle des moyens de se défendre contre la chaleur estivale représentée alors comme la furie du lion de Némée ${ }^{27}$. Cependant, les conseils de l'Arétin pour y remédier laissent penser que le sujet est en fait plus complexe et que les formulations employées peuvent avoir un sens figuré. En effet, la chaleur pourrait faire référence à des désirs nocifs, à des vices ou encore à des pulsions pécheresses. Pétrarque reprend la métaphore et rédige une réponse supposée servir de remède aux inconvénients énoncés. Il esquisse

\footnotetext{
${ }^{26}$ Voir Paul Gavrilyuk et Sarah Coakley, The Spiritual Senses. Perceiving God in Western Christianity, New York/Melbourne/Madrid et al, Cambridge University Press, 2012.

27 «Queris ex me, seu per te querit amicus maior, imo quidem, ut intelligenti datur, ambo queritis, quid remedii, norim advers us Nemei rabiem Leonis tot nunc impetu Phebicrines ac faciem accendentis », Francesco Pétrarque, Epistolae de rebus familiaribus et variae, ed. J. Fracassetti, Florentiae, Typis Felicis Le Monnier, 18591863 , t. III, p. 440.
} 
ainsi la figure d'un grand arbre vert, situé dans un lieu alpin, agréable et difficile d'accès. Sa description est ensuite beaucoup plus analytique et didactique : «je vous le décrirai avec les signes qui le caractérisent, pour que vous puissiez le reconnaître et que vous ne soyez pas trompés par une quelconque ressemblance ${ }^{28} \gg$. Il évoque alors quatre branches, dont les ombres sont utilisées par des bergers pour s'y reposer. Alors que ces derniers sont de plus en plus nombreux, un agriculteur descend du ciel pour arroser l'arbre, entraînant la naissance de nouvelles branches qui se couvrent d'un feuillage toujours plus dense. Dès lors, l'arbre compte sept branches: si les quatre premières retombent vers la terre, les trois nouvelles se dressent vers le ciel. Le poète d'Arezzo souligne ensuite, avec une extrême précision, les éléments qui distinguent cet arbre des autres : en effet, des oiseaux viennent se poser sur le végétal, chanter et y faire leur nid, des pommes très sucrées mûrissent sur les rameaux, un tapis d'herbe verte et de fleurs rouges recouvre le sol, enfin, une source d'eau claire bruisse doucement sous l'ombre du conifère ${ }^{29}$. Pétrarque termine sa lettre par une mise en garde sur le comportement que le lecteur doit avoir face à cette image : «Voilà donc mon conseil : cherchez cet arbre de toutes vos forces, et quand vous l'aurez trouvé, serrez-le

28 «Quam ut nosse possitis, neu similitudine aliqua forte fallamini, signis hanc describam suis », Ibid., p. 441.

${ }^{29}$ «Quatuor tantum haec olim ramos habuit, dum illam fessi operum pastores avidus frequentarent. Venit incognitus coelestis agricola, et sarculo cultam pio, serotinique roris suavitate conspersam ramis auxit ac frondibus nimium. Hodie septem habet, quorum inferiores quatuor tellurem, tres altissimi coelum spectant: ramuli autem plures sunt de quibus nunc loquendi non est locus. Illic assidue felices aurae circumsibilant, canorae simul et candidae volucres nidificant, poma praedulcia divites ramos premunt. Mite solum, herbae variae et purpurei flores tegunt : in quibus levis accubitus, odor suavissimus, et ad se oculos trahens color. Fons ad umbram nitidus amoenitate lymphraum scatebrisque perennibus manat, cuius in circuitu roscidis cespitibus ripisque recentibus cursum fraenantibus, undarum late gratissimum murmur strepit ». Ibid., p. 441. 
avidement dans vos bras, prenez soin de lui, aimez-le $\mathrm{e}^{30} \gg$. Ainsi à la fin de sa lettre, le poète recommande une série d'activités mentales qui ne peuvent être effectuées qu'en reconstruisant l'image de l'arbre dans son esprit.

La réponse des deux amis se devine en substance dans une autre lettre du poète arétin, écrite entre le mois d'octobre et celui de novembre de la même année. Leur réaction semble être double. Les amis demandent à Pétrarque de leur fournir l'interprétation de cet arbre tout en tentant de participer à la réflexion : Giovanni et Lucchino lui auraient ainsi envoyé deux images «magnifiquement colorées » qui essaient d'interpréter le texte. Les deux amis semblent demander à Pétrarque la validation ou la correction de cette exégèse à travers ces images. Lucchino del Verme dessine un arbre qui est la représentation graphique de ce que le poète avait décrit. À côté de ce dessin, Giovanni Fedolfi peint son contraire, c'est-à-dire « le sable de cette vie qui est l'emplacement des mortels, où temporellement une chose et l'autre sont confondues sans égales proportions, et ce jusqu'à la fin des temps, quand le sable sera trié et dispersé par le souffle du juge suprême ${ }^{31} »$. La fin de la lettre révèle que cette seconde image, qui accompagne la première représentation classique de l'arbre, est celle d'un globe terrestre désertique. Dans sa réponse, Pétrarque souligne d'abord l'habileté et la justesse dont ses amis ont fait preuve en reconnaissant le type d'arbre qu'il avait dessiné en paroles, à savoir l'arbre des vertus. Ensuite, il donne une analyse méticuleuse de chacun des éléments - signa - de cette représentation : quatre branches pour les quatre vertus cardinales cultivées par les

\footnotetext{
30 «Haec igitur summa consilii mei est. Arborem hanc quaerite omni studio ut facitis; inventam cupidis ulnis arripite, et tenete, et colite, et amate $(30,42) \gg$ Ibid.

31 "Quod auribus ingesseram oculis subiecisti, non contentus nisi et eius oppositum insuper, et huius vitae arenam habitatam mortalibus addidisses: ubi ad tempus utrumque permixtim, et heu! Non aequis portionibus confusa sunt omnia, discernenda novissime, et supremi flabro iudicii ventilanda $(31,7-11) »$, p. 473.
} 
anciens, c'est-à-dire les bergers qui s'étaient réfugiés dans son ombre ; les trois branches-vertus théologales; le Christ-agriculteur qui descend pour creuser et arroser la terre avec son sang rédempteur. Pétrarque attire l'attention sur la fonction protectrice de l'arbre, consistant à préserver l'âme des passions humaines qui la perturbent. Dans cette seconde lettre, il loue l'extrême fécondité que son texte a générée : «d'une petite semence, j'ai obtenu une récolte copieuse ${ }^{32} \gg$. Cette stratégie répond parfaitement à la logique d'ordre et de classification que nous essayons d'illustrer ici et qui semble être un mécanisme cognitif courant dans la littérature de cette époque. Plus précisément, nous voyons, dans ce cas particulier, le motif de l'arbor comme image puissante qui participe à la captatio mentis et, en même temps, une figure taxinomique qui est intégrée dans l'écriture ${ }^{33}$. Elle développe également l'activité herméneutique et productrice comme si l'organisation et la classification poussaient le lecteur à déployer une espèce d'inertie scripturaire, c'est-àdire, à développer l'acte d'écriture et de réflexion. En effet, la traduction verbale de l'arbre des vertus de Pétrarque a généré la représentation de son contraire. Cependant, et à l'inverse des textes de Marguerite d'Oingt, il ne s'agit pas d'une opposition symétrique représentée par l'arbre des vices : Pétrarque utilise la métaphore du désert sous la forme du globe terrestre $^{34}$. Ainsi, cette invention a non seulement permis aux interlocuteurs de reconnaître l'arbre des vertus, mais également de pousser le jeu herméneutique en utilisant une manière créative de montrer

\footnotetext{
32 «Uberem messem parvo semine messui », Ibid., p. 473.

${ }^{33}$ Lina Bolzoni, La rete delle immagini. Torino, Einaudi, 2002, p. 110.

${ }^{34}$ En fait, la mémoire apparaît souvent dans la culture antique et médiévale comme une matière informe, une forêt ou un désert qu'il faut ordonner. Les parties de l'arbre, les racines, le tronc ou les branches, distribuent les différente lieux - loci-qui ordonnent les images ou notions qu'il faut mémoriser. Mary Carruthers, The Book of Memory, op. cit., p. 39, 78, 324.
} 
le contraire de l'arbre ${ }^{35}$. Pétrarque se prend lui aussi au jeu et décrit l'image reçue, avec un grand nombre de termes associés au désordre, à la précarité, au mélange chaotique. Le globe terrestre est bien un troisième modèle servant à ordonner la pensée à partir des deux premières images évoquées par l'auteur et les lecteurs. Nous sommes donc ici devant un texte qui propose une dispositio visuelle, une classification des contenus à travers une arborescence taxinomique, en forme d'arbre, reconnue par les lecteurs. Ces derniers distribuent mentalement ou physiquement - mais en tout cas, visuellement - les signa, ce qui les pousse à comprendre l'allégorie et à créer son contraire suivant, de manière consciente ou non, les motifs iconographiques basés sur la symétrie et typiques de l'époque, comme l'arbor bona/arbor mala.

Les deux cas évoqués soulèvent donc de nombreuses hypothèses portant sur les schémas d'écriture comme mises en ordre du savoir. Pourtant, ces hypothèses suscitent également plusieurs interrogations. En guise de conclusion, nous proposons quatre points qui soulignent, à nos yeux, l'intérêt des analyses sur ce sujet. D'abord, ces deux cas montrent clairement le rapport entre modèles d'écriture et de visualisation graphique. En ce sens, il nous semble pertinent de considérer, dans l'étude des écrits du début du XIV ${ }^{\mathrm{e}}$ siècle, que les structures arborescentes sont des organisations du savoir immanentes au texte. Dans certains cas, comme ceux que nous avons analysés ici, il s'agit de moyens de visualisation ou de spatialisation qui ont un caractère arborescent, c'est-àdire que la figure de l'arbre y est explicite. Dans d'autres cas, néanmoins,

\footnotetext{
${ }^{35}$ Lina Bolzoni, op. cit., p. 111.
} 
l'arborescence est schématique et dépouillée d'analogies végétales, sans que cela implique une interprétation contraire ${ }^{36}$.

Ensuite, force est de noter que l'inversion et la symétrie sont les deux composantes organisatrices qui articulent l'activité combinatoire, déclenchant une action à la fois herméneutique et productrice. Il faut sans doute être prudent sur la formulation d'une telle hypothèse, mais le nombre de textes faisant appel à des structures arborescentes et à des variantes de ce modèle est important : ce pourrait donc être une piste à développer, pour comprendre le succès du motif de l'arbor pendant le Moyen Âge. Cette première ébauche voudrait être une réflexion pour appréhender ces figures végétales au moyen d'une analyse plus exhaustive d'une part, et d'autre part, à travers la recherche d'une méthodologie précise. L'étude de Marco Rainini montre bien l'intérêt d'une telle approche: pour lui ce genre de structures accorde un rôle fondamental aux éléments comptables, mais aussi au rythme qui organise les diagrammes ${ }^{37}$. Ainsi, la correspondance symétrique de parallélisme ou d'opposition évoquée dans ces exemples pourrait être un des rythmes de la création littéraire et de l'exégèse-que nous pourrions appeler «spatialisation rythmique »-. En fait, les arbores, tout comme les figures des roues, montrent la recherche d'un ordre et d'une ratio dans une image synthétique. C'est dans cette recherche que le rythme impose une figure en raison du contenu qui doit être visualisé. L'arborescence, avec sa dialectique unité-diversité et sa morphologie symétrique, est une

\footnotetext{
${ }^{36}$ Marco Rainini, «L'ordre del desordre i els seus ordenaments. Ratio i vida espiritual en els diagrames dels vicis del segle XII » dans El monestir interior, dir. Victoria Cirlot et Blanca Garí, Barcelona, Fragmenta, 2017, p. 39-77, ici p. 60-61.

${ }^{37}$ Ibid., p. 41. Le rythme, ici conçu symboliquement: Jean-Claude Schmitt, «A history of rhythms during the Middle Ages », The Medieval History Journal, vol. 15, 2012, p. 1-24.
} 
figure susceptible d'être utilisée dans un rythme de correspondance dichotomique ${ }^{38}$.

En troisième lieu, il est nécessaire de mettre en évidence le lien entre cette pratique d'organisation productive du savoir et la mnémotechnie, une science qui a été oubliée pendant longtemps dans les études de la culture du Moyen Âge, mais qui semble prendre un poids important dans les dernières études concernant les domaines de l'art, la littérature, la philosophie ou la spiritualité ${ }^{39}$. Pourtant, hiérarchiser, c'està-dire diviser et ordonner, sont les deux lignes directrices des techniques de mémorisation depuis l'Antiquité. Comme l'a démontré Mary Carruthers, la mémoire a, pendant le Moyen Âge, une fonction cognitive bien complexe qui englobe l'activité reproductrice, mais aussi productrice $^{40}$. Ainsi, à l'époque médiévale, la conception de l'imagination et celle de la créativité pourraient bien avoir des fonctions et des activités liées à la mémoire ; ces dernières s'emploieraient selon un principe de correspondance, d'analogie ou encore, de combinaison ${ }^{41}$. Par conséquent, ces arguments amènent à envisager une conception de la création liée à

\footnotetext{
${ }^{38}$ Marco Rainini, op. cit., p. 67-68.

${ }^{39}$ Il faut notamment citer le projet Trames Arborescentes. Modalités, caractéristiques et efficacité de dispositifs organisationnels en arbre ou en arborescence, qui depuis 2015 s'est focalisé sur ces aspects d'une manière transdisciplinaire et transhistorique mais en ayant un accent particulier sur le Moyen Âge tardif. https://trarbor.hypotheses.org/

40 «The monastic memorative arts are what made invention and interiority possible; they were ultimately prospective, not retrospective, productive, not reproductive », Jeffrey Hamburger, « Reviews work: The Craft of Thought: Meditation, Rhetoric, and the Making of images, 400-1200, Cambridge Studies in Medieval Literature 34 by Mary Carruthers », Medium Aevum, vol. 68, n 2, 1999, p. 307-309, ici p. 307.

${ }^{41}$ Selon Sylvia Huot, ces figures mnémotechniques englobaient «the reception, processing, storing, retrieval, and recombination of material gleaned from reading in its largest sense: attention to the texts, images, and teachings offered by the world at large ». Sylvia Huot, «Inventional Mnemonics Reading and Prayer: A Reply to Mary Carruthers », Connotations vol. 3, 1993-1994, p. 103-109, cit. p. 109.
} 
l'organisation, à la spatialisation et à la combinaison du savoir déjà acquis $^{42}$.

Enfin, bien que les mécanismes de création et de transmission du savoir soient difficiles à étudier, il semble que la compréhension de la conception historique de ces outils de création soit, en revanche, plus accessible. Cette approche permet ainsi de retracer un réseau de pratiques cognitives qui, dans le domaine de la littérature et de l'histoire, a presque toujours été envisagé par le contenu et le support matériel de transmission et non par les formes contentant le savoir. Si les deux cas étudiés appartiennent à des milieux cultivés - d'un côté monastique, d'un autre humaniste - et sont donc issus de la littérature didactique et méditative généralement riche de ce genre de procédés, le caractère implicite de ces derniers au sein de ces deux exemples de textes privés suggère l'importance dans la culture médiévale de ces outils, encore bien souvent inconnus. En effet, au-delà des contenus parfois savants, les contenants et les formes ici analysés pourraient être plus diffusés dans l'ensemble de la culture médiévale. De la même manière, Marco Rainini en comparant les arborescences de Conrad d'Hirsau et de Pierre de Potiers, a montré une certaine similitude dans leur spatialisation rythmique quand bien même les schémas proviennent de mouvements traditionnellement différenciés. Se fondant sur l'analyse de ces images, il propose de nuancer la division rigide entre attitudes monastiques et scolastiques. Selon lui, le monde des écoles urbaines et celui des monastères seraient finalement moins distants que ce qui a souvent été présenté ${ }^{43}$.

\footnotetext{
${ }^{42}$ Mary Carruthers, The Craft of Thought, op. cit., p. 12.

43 «Les imatges que hem considerat, per la manera com s'organitzen, per les seves fonts $i$, en definitiva, almenys en part, també per la seva transmissió, em sembla que no justifiquen una divisió gaire rígida entre actituds monàstiques $i$ actituds escolàstiques [...] El món de les escoles urbanes $i$ catedralícies $i$ el de les monàstiques, en definitiva, ens apareixen [...] molt menys allunyats entre si del que sovint se'ns han presentat ». Marco Rainini, op. cit., p. 65, 67.
} 
En étudiant la circulation et les modalités de diffusion du savoir utilisé, une autre carte de migrations pourrait être dessinée. Cette carte ne serait pas seulement définie à partir des contenus des textes ou des images, mais aussi par la circulation de ces outils visuels qui structurent le savoir. De cette façon, les fractures constatées entre culture savante et culture populaire, entre savoir masculin et savoir féminin, ou encore entre Europe du Nord et Europe du Sud pourraient être nuancées grâce à la recherche d'une appartenance culturelle basée sur un réseau d'usages du savoir et non sur les idées. 


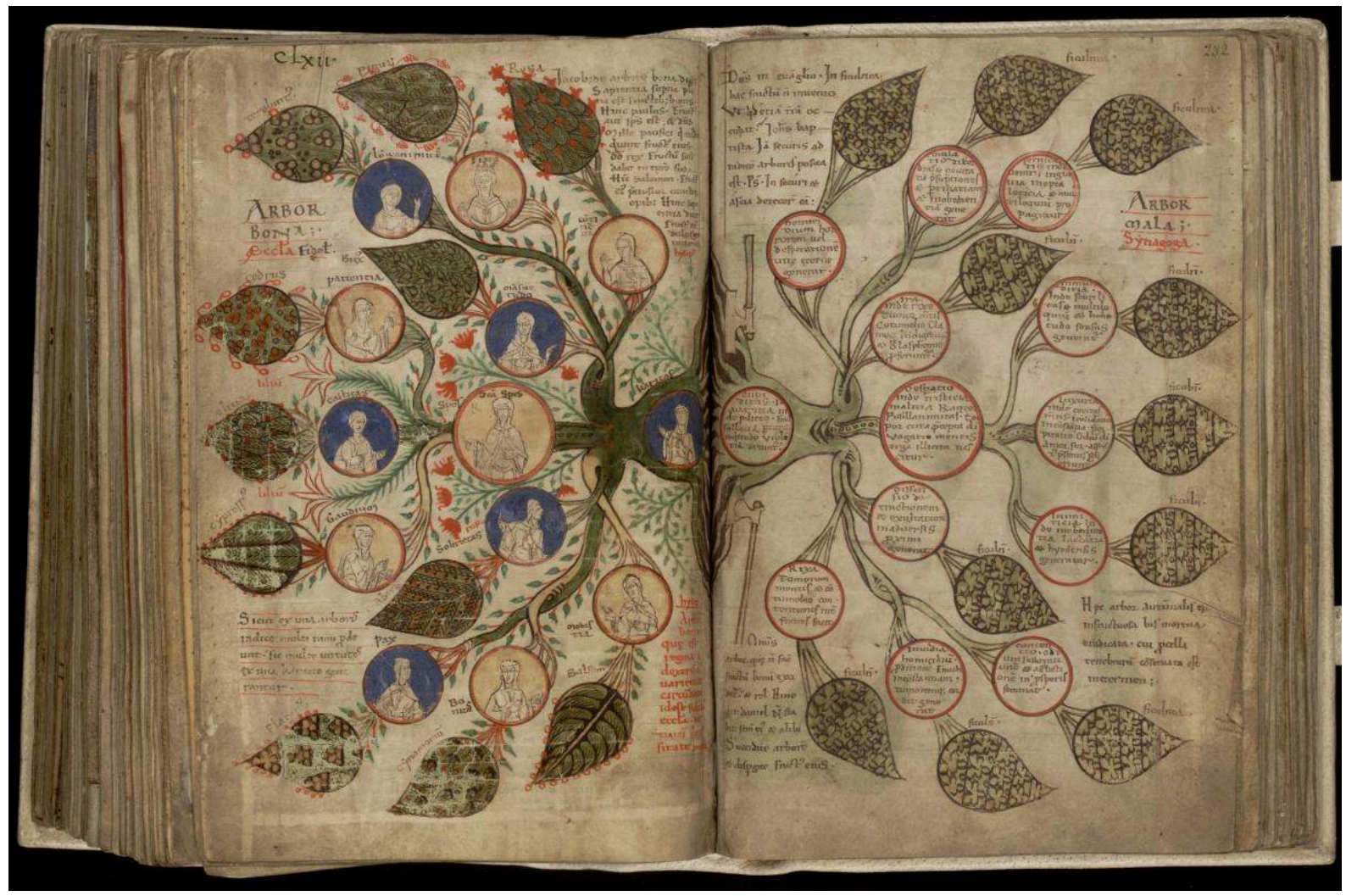

Fig. 1. Arbor bona et arbor mala, V.1121. Liber Floridus de LAMBert de SAint-OMER, MS 92, FOL. 231V-232R. UNIVERSITEITSBIBLIOTEECK, GENT. 